\title{
Study on Linear Combination of Long Memory Processes Corrupted by Additive Noises for fMRI Time Series Analysis
}

\author{
Wonsang You and Catherine Limperopoulos \\ Division of Diagnostic Imaging and Radiology, \\ Children's National Health System, \\ 111 Michigan Ave, NW, Washington, DC. \\ wyou@childrensnational.org \\ http://www.childrensnational.org
}

\begin{abstract}
Estimating the long memory parameter of the fMRI time series enables us to understand the fractal behavior of neural activity of the brain through fMRI time series. However, the existence of white noise and physiological noise compounds which also have fractal properties prevent us from making the estimation precise. As basic strategies to overcome noises, we address how to estimate the long memory parameter in the presence of additive noises, and how to estimate the long memory parameters of linearly combined long memory processes.
\end{abstract}

Keywords: long memory process, fractional Brownian motion, fMRI, resting state

\section{Introduction}

Fractal can be regarded as self-similarity of an object which means that the statistical properties remain invariant throughout different scales. It is well known that many natural phenomena have such a fractal structure; for example, it can be found at river networks, clouds, trees, neuronal networks, Koch curve, and so forth [11]. The fMRI time series have also fractal properties or $1 / f$ spectral densities [3][12]. Thus, some neuroscientists have attempted to study fractal properties of fMRI time series by estimating the fractal dimension or long memory parameter. In results, they have observed not only that the fMRI time series tend to be corrupted by a fractional Gaussian noise (fGn) which has fractal properties, but also that the Hurst exponent of the fGn noise provides valuable information on Alzheimer's disease [12], Moreover, the fractal properties are stronger in grey matter which has higher population of neurons, than in white matter of the brain even during resting state [4]. These reports indicate that the fractal properties of fMRI time series may represent neural activity of the brain. Thus, it is manifest that the fractal properties are one of important features to analyze fMRI time series.

Our interest is to estimate the long memory parameter of the endogenous signal which can be modeled as a long memory process in order to track the 
spontaneous neural activity of the brain during resting state of a subject through analyzing fMRI time series. Unfortunately, the data normally tend to suffer from tremendous noise compounds such as cardiac pulse, respiration, subject motion, and scanner noise. While it is not serious in task-based experiments, it could be a critical problem in resting state fMRI analysis since it may significantly prevent us from extracting the endogenous signal which arises from neural activity of the brain. It means that the long memory behavior of a raw fMRI time series may be attributed not only to endogenous neural activity but also to physiological noise, subject movement, and other sources [13][14]. To precisely estimate the long memory parameter of a pure endogenous signal, we should model the raw fMRI time series as the linear sum of several long memory processes (for example, the summation of endogenous signal and physiological pulsations) and additive noises. Under this model, our works consist of two steps: (1) estimating the parameters of each component in the model, and (2) classifying each component into endogenous signal, physiological noises, or other additive noises.

Here, we focus on the first step: estimating the parameters of linearly combined long memory processes in the presence of additive noises. To be specific, we address two topics; one is the parameter estimation of a long memory process in the presence of additive noises, and the other is the parameter estimation of linearly combined long memory processes. Regarding the former topic, we thought that Achard's method for a fractional Brownian motion corrupted by additive noises [7] can be also applicable to estimate the parameter of a long memory process corrupted by additive noise. In the latter topic, we will introduce a method to estimate the parameters of linearly combined long memory processes. The fact that the linear combination of long memory processes results in the linear combination of wavelet variances allows us to use multiexponential analysis in order to estimate the parameters of linearly combined long memory processes.

\section{The Signal Model}

Let $\mathbf{Y}:=\left\{\mathbf{Y}_{l}\right\}_{l=1, \cdots, L}$ be a set of $L$ stochastic processes where $\mathbf{Y}_{l}:=\left\{Y_{l}(t)\right\}_{t=1, \cdots, N}$ for $l=1, \cdots, L$. In other words, we have $L$ fMRI time series with $N$ time points. We assume that each process can be modeled as the linear combination of endogenous signal, cardiac noise, respiratory noise, and white noise as follows

$$
\mathbf{Y}_{l}=\mathbf{X}_{l}+\mathbf{C}_{l}+\mathbf{R}_{l}+\mathbf{N}_{l}
$$

where $\mathbf{X}_{l}:=\left\{X_{l}(t)\right\}_{t=1, \cdots, N}$ is an endogenous signal at $l$-th voxel, $\mathbf{C}_{l}:=\left\{C_{l}(t)\right\}_{t=1, \cdots, N}$ is a cardiac noise at $l$-th voxel, $\mathbf{R}_{l}:=\left\{R_{l}(t)\right\}_{t=1, \cdots, N}$ is a respiratory noise at $l$-th voxel, and $\mathbf{N}_{l}:=\left\{N_{l}(t)\right\}_{t=1, \cdots, N}$ is an additive white Gaussian noise at $l$-th voxel. That is, $N_{l}(t)$ for $t=1, \cdots, N$ are i.i.d. Gaussian random variable with variance $\sigma_{l}^{2}$. Since the first step is to eliminate the additive white noise $\mathbf{N}_{l}$, we can simplify the above equation into

$$
\mathbf{Y}_{l}=\dot{\mathbf{X}}_{l}+\mathbf{N}_{l}
$$

where $\dot{\mathbf{X}}_{l}=\mathbf{X}_{l}+\mathbf{C}_{l}+\mathbf{R}_{l}$. 


\section{Parameter Estimation of a Long Memory Process Corrupted by Additive Noises}

\subsection{Some Definitions}

As we discussed in the previous section, the endogenous signal $\mathbf{X}_{l}$ can be modeled as a type of long memory process. Likewise, we can also regard cardiac noise $\mathbf{C}_{l}$ and respiratory noise $\mathbf{R}_{l}$ as long memory processes. Indeed, there have been a lot of evidences which imply that heartbeat [16][17][18][19][20][21] and respiration $[22][23][24]$ also have fractal properties.

We suppose that the linear summation of long memory processes is approximately a long memory process. In other words, if each process $X_{k}$ for $k=1, \cdots, N$ has long memory, the process

$$
Z(t):=\sum_{k=1}^{N} \alpha_{k} X_{k}(t)
$$

also has long memory for $\alpha_{k} \in \mathbb{R}$. We did not prove this hypothesis but we will indirectly see at the section 4 how reasonable it is. In addition, Christoph Thäle [25] proved that the linear combination of $N$ fractional Brownian motions with $H_{k}>1 / 2$ for $k=1, \cdots, N$ is also long-range dependent. Under this hypothesis, $\dot{\mathbf{X}}_{l}$ in $(2)$ can also be regarded as a long memory process.

We adopt the definition of a long memory process suggested by Moulines et al. [5]; that is, a real-valued discrete process $\dot{\mathbf{X}}_{l}$ is said to have memory parameter $d_{l}$ (and is called a $M\left(d_{l}\right)$ process) if, for any integer $D>d_{l}-1 / 2$, the $D$-th order difference process $\Delta^{D} \dot{\mathbf{X}}_{l}\left(\right.$ where $\Delta^{D} \dot{X}_{l}(k):=\Delta^{D-1} \dot{X}_{l}(k)-\Delta^{D-1} \dot{X}_{l}(k-1)$ ) is weakly stationary with spectral density function

$$
S_{\Delta^{D} \dot{\mathbf{X}}_{l}}(f):=\left|1-e^{-i 2 \pi f}\right|^{2\left(D-d_{l}\right)} S_{\dot{\mathbf{X}}_{l}}^{*}(f), \quad f \in(-1 / 2,1 / 2)
$$

where $S_{\dot{\mathbf{x}}_{l}}^{*}(f)$ is a non-negative symmetric function which is bounded on $(-1 / 2,1 / 2)$.

To estimate the long memory parameter $d_{l}$, let us define a filter $a$ of length $p+1$ satisfying the following conditions ${ }^{1}$ :

1. $\sum_{k=1}^{n} a_{k}^{2}=1$.

2. There exists $\alpha>0$ such that $\sup _{k \in[0, n)}\left|\hat{a}_{k}\right|\left(1+\left|\frac{k}{n}\right|\right)^{\alpha}<\infty$ where $\hat{a}_{k}:=$ $\sum_{q=1}^{n} a_{q} e^{-i 2 \pi q k / n}$.

3. It has $M$ vanishing moments, i.e. $\sum_{q=0}^{p} q^{j} a_{q}=0$ for $j=0, \cdots, M-1$, and $\sum_{q=0}^{p} q^{M} a_{q} \neq 0$.

\footnotetext{
${ }^{1}$ The notation of filter was adopted from Achard et al. [7], but its conditions are identical to those of the wavelet filter defined by Moulines et al. [5]. The only difference is that the conditions are based on Discrete Fourier Transform while Moulines et al.'s filter conditions are based on Continuous Fourier Transform. We just assume, without proof, that Moulines et al.'s theories are valid even in our conditions of the wavelet filter.
} 
On the other hand, the dilated filter ${ }^{2} a^{j}$ is defined as the filter of length $2^{j} p+1$ such that for $j \geq 0$ and $k=0, \cdots, 2^{j} p$,

$$
a_{k}^{j}= \begin{cases}a_{k / 2^{j}} & \text { if } k / 2^{j} \text { is an integer } \\ 0 & \text { otherwise. }\end{cases}
$$

Let $\dot{\mathbf{X}}_{l}^{a}$ be the vector $\dot{\mathbf{X}}_{l}$ filtered with $a$ such that for $i=l+1, \cdots, n$

$$
\dot{X}_{l}^{a}(i):=\sum_{q=0}^{l} a_{q} \dot{X}_{l}(i-q) .
$$

\subsection{Asymptotic Approximation of Spectral Density}

Moulines et al. showed that the spectral density of the wavelet coefficients of an $M(d)$ process can be asymptotically approximated by that of fractional Brownian motion (FBM) if the memory process satisfies some conditions [5]. If the process $\dot{\mathbf{X}}_{l}$ is covariance stationary and have the spectral density

$$
S_{\dot{\mathbf{X}}_{l}}(f)=\left|1-e^{-i 2 \pi f}\right|^{-2 d} S_{\dot{\mathbf{X}}_{l}}^{*}(f)
$$

for $0<d<1 / 2, X, \dot{\mathbf{X}}_{l}$ is said to have long memory, and $S_{\dot{\mathbf{X}}_{l}}(f)$ is called a generalized spectral density [26]. On the other hand, let $\mathcal{H}(\beta, L)$ be the set of even non-negative functions $g$ on $[-1 / 2,1 / 2]$ such that

$$
|g(f)-g(0)| \leq L g(0)|2 \pi f|^{\beta} .
$$

Theorem 1. Given the constants $L, \beta$ such that $0<L<\infty, \beta \in(0,2]$, and $\left[d_{\text {min }}, d_{\text {max }}\right] \subset((1+\beta) / 2-\alpha, M+1 / 2)$, there exists a constant $C>0$ such that, for all $j \geq 0, d_{l} \in\left[d_{\min }, d_{\max }\right]$ and $S_{\mathbf{\mathbf { X }}_{l}}^{*} \in \mathcal{H}(\beta, L)$,

$$
\left|\nu_{l}^{2}(j)-S_{\dot{\mathbf{X}}_{l}}^{*}(0) K\left(d_{l}, a\right) 2^{2 d_{l} j}\right| \leq C S_{\dot{\mathbf{X}}_{l}}^{*}(0) L 2^{\left(2 d_{l}-\beta\right) j}
$$

where

$$
\begin{aligned}
\nu_{l}^{2}(j) & :=\operatorname{Var}\left(\dot{\mathbf{X}}_{l}^{a^{j}}\right) \text { and } \\
K\left(d_{l}, a\right) & :=\sum_{k=0}^{n-1}\left|\frac{k}{n}\right|^{-2 d_{l}}\left|\hat{a}_{k}\right|^{2} .
\end{aligned}
$$

The theorem was proved by Moulines et al. in [5]. They showed that $S_{\mathbf{X}_{l}}^{*}(0) K\left(d_{l}, a\right) 2^{2 d_{l} j}$ is the spectral density of the filtered series of a generalized FBM $B_{(d)}$ which is defined as a mean-zero Gaussian process with covariance

$$
\operatorname{Cov}\left(B_{(d)}^{a^{j_{1}}}, B_{(d)}^{a^{j_{2}}}\right)=\sum_{k=0}^{n-1}|2 \pi k|^{-2 d} \hat{a}_{k}^{j_{1}} \hat{a}_{k}^{j_{2}}
$$

\footnotetext{
${ }^{2}$ Notice that the notation $a^{j}$ is identical to $a^{2^{j}}$ in Achard et al.'s definition [7].
} 
for $d \in(1 / 2-\alpha, M+1 / 2)$. Therefore, from $(9), \nu_{l}^{2}(j)$ can be well approximated by $S_{\mathbf{X}_{l}}^{*}(0) K\left(d_{l}, a\right) 2^{2 d j} ;$ i.e.

$$
\nu_{l}^{2}(j) \approx S_{\dot{\mathbf{X}}_{l}}^{*}(0) K\left(d_{l}, a\right) 2^{2 d j}
$$

if $d_{l}<\beta / 2$. By denoting $\gamma_{l}:=S_{\mathbf{\mathbf { x }}_{l}}^{*}(0) K\left(d_{l}, a\right)$, we have the following regression model

$$
\log \left(\nu_{l}^{2}(j)\right)=2 d_{l} j+\log \left(\gamma_{l}\right) .
$$

Since $\nu_{l}^{2}(j)$ can be estimated by the empirical variance $\hat{\nu}_{l}^{2}(j)$, we can also obtain estimator of the memory parameter $d$ through the ordinary regression estimator

$$
\hat{d}_{l}:=\frac{\mathbf{w}^{T}}{2\|\mathbf{w}\|^{2}}\left(\log \left(\hat{\nu}_{l}^{2}(j)\right)\right)_{j=J_{1}, \cdots, J_{2}}
$$

where the vector $\mathbf{w}:=\left[w_{0}, \cdots, w_{l}\right]^{T}$ satisfies $w_{i}=j-\frac{1}{J_{2}-J_{1}+1} \sum_{j=J_{1}}^{J_{2}} j$. From (14) and (15), we have $\hat{d}_{l}$ and $S_{\dot{\mathbf{X}}_{l}}^{*}(0)$.

This least squares estimator of long memory parameter is similar with that of fractional Brownian motion discussed by Coeurjolly and Achard [27][7]. For example, they described that a fractional Brownian motion $\mathbf{B}_{H}$ with Hurst parameter $H \in(0,1)$ and scaling coefficient $C>0$ has the following property

$$
E\left(\overline{\left(\mathbf{B}_{H}^{a^{j}}\right)^{2}}\right)=2^{2 H j} C^{2} \pi_{H}^{a}(0)
$$

where $\overline{\left(\mathbf{B}_{H}^{a^{j}}\right)^{2}}$ denotes the empirical mean of the squared filtered coefficients, and

$$
\pi_{H}^{a}(i)=-\frac{1}{2} \sum_{q, r=0}^{l} a_{q} a_{r}|q-r+i|^{2 H} .
$$

The equation (16) of a FBM is similar with the equation (13) of a long memory process, which indicates that Achard et al.'s method [7] for estimating the Hurst parameter of a fractional Brownian motion in the presence of additive noise is applicable to the general class of long memory processes without any modification. In the next sections 3.3 and 3.4, we will summarize how to apply Achard et al.'s method to general long memory processes.

\subsection{Model B0: Long Memory Process with an Additive Brownian Motion}

Let us consider the following model

$$
\mathbf{Y}_{l}(t)=\dot{\mathbf{X}}_{l}(t)+\sigma_{l} \mathbf{B}^{(0)}(t)
$$

where $\sigma>0, \dot{\mathbf{X}}_{l}(t)$ is a long memory process with parameter $d_{l}$ as defined in the section 3 and $\mathbf{B}^{(0)}(t)$ for $t=1, \cdots, N$ is a standard Brownian motion. Then, from (13), the variance of the filtered series of $\mathbf{Y}_{l}$ will be

$$
\mathbf{E}\left(\overline{\left(\mathbf{Y}^{a^{j}}\right)^{2}}\right)=2^{2 d_{l} j} \gamma_{l}+2^{j} \sigma_{l}^{2} \pi_{1 / 2}^{a}(0)
$$


where $\gamma_{l}=S_{\dot{\mathbf{x}}_{l}}^{*}(0) K\left(d_{l}, a\right)$. Thus, we can apply the same method as Achard et al. did as follows; if we define $\mathbf{Z}^{a^{j}}(i)=\frac{\mathbf{Y}^{a^{j}}(i)}{2^{j / 2}}$, the least squares estimate is computed by

$$
\hat{d}^{B 0-S T}=\frac{1}{2}+\frac{\mathbf{w}^{T}}{2\|\mathbf{w}\|^{2}}\left(\log \left(\overline{\left(\mathbf{Z}^{a^{j+1}}\right)^{2}}-\overline{\left(\mathbf{Z}^{a^{j}}\right)^{2}}\right)\right)_{j=J_{1}, \cdots, J_{2}}
$$

\subsection{Model B1: Long Memory Process with an Additive White Noise}

Let us consider the following model

$$
\mathbf{Y}_{l}(t)=\dot{\mathbf{X}}_{l}(t)+\sigma_{l} \mathbf{B}^{(1)}(t)
$$

where $\sigma_{l}>0$ and $\mathbf{B}^{(1)}(t)$ for $t=1, \cdots, N$ are i.i.d. standard Gaussian variables. According to Achard et al. [7],

$$
\mathbf{E}\left(\overline{\left(\mathbf{Y}^{a^{j}}\right)^{2}}\right)=2^{2 d_{l} j} \gamma_{l}+\sigma_{l}^{2}|a|^{2}
$$

and the least squares estimate is

$$
\hat{d}^{B 0-S T}=\frac{\mathbf{w}^{T}}{2\|\mathbf{w}\|^{2}}\left(\log \left(\overline{\left(\mathbf{Y}^{a^{j+1}}\right)^{2}}-\overline{\left(\mathbf{Y}^{a^{j}}\right)^{2}}\right)\right)_{j=J_{1}, \cdots, J_{2}}
$$

\section{Linear Combination of Lone Memory Processes}

In the previous section, we dealt with two models: a fractional Brownian motion contaminated by a standard Brownian motion, and a fractional Brownian motion contaminated by an additive white noise. Here, we will generalize the signal model; let us consider the following model

$$
\mathbf{Y}_{l}(t)=\sum_{k=1}^{K} \dot{\mathbf{X}}_{l, k}(t)+\sigma_{l} \mathbf{B}^{(1)}(t)
$$

where $K$ is the unknown integer, $\dot{\mathbf{X}}_{l, k}(t)$ for $k=1, \cdots, K$ is a long memory process with parameter $d_{l, k}$ and $\mathbf{B}^{(1)}(t)$ for $t=1, \cdots, N$ are i.i.d. standard Gaussian variables. The variance of the filtered series of $\mathbf{Y}_{l}$ will be

$$
\begin{aligned}
\mathbf{E}\left(\overline{\left(\mathbf{Y}_{l}^{a^{j}}\right)^{2}}\right) & =\sum_{k=1}^{K} 2^{2 d_{l, k} j} \gamma_{l, k}+\sigma_{l}^{2}|a|^{2} \\
& =\sum_{k=1}^{K} e^{2 \log (2) d_{l, k} j} \gamma_{l, k}+\sigma_{l}^{2}|a|^{2}
\end{aligned}
$$


where $\gamma_{l, k}=S_{\dot{\mathbf{X}}_{l, k}}^{*}(0) K\left(d_{l, k}, a\right)$. Then, we would like to estimate $K$ and $d_{l, k}$ for all $k=1, \cdots, K$ given the measurement $\mathbf{E}\left(\overline{\left(\mathbf{Y}^{a^{j}}\right)^{2}}\right)$ for $j=J_{1}, \cdots, J_{2}$.

This problem can be interpreted as the multiexponential analysis which is a common problem in the various fields such as physics, chemistry, medical imaging, and so forth. There exist numerous methods such as nonlinear least squares analysis (grid search, gradient search, Gauss-Newton method, Levenberg-Marquard method) [1] and Bayesian probability theory [2]. Even though in general the solution of this problem is not unique [1], it can be converted to the well-posed problem by applying some constraints on parameters.

The other issue is that the estimation is sensitive to the number of scales $J_{2}-J_{1}+1$ and the resolution of parameters. In otherwords, the decrease in the number of scales and the increase in the number of components $K$ or the resolution $d_{l, k_{1}} / d_{l, k_{2}}$ (if $d_{l, k_{1}}>d_{l, k_{2}}$ for $k_{1}, k_{2}=1, \cdots, K$ ) make the parameter estimation less precise. In the fMRI analysis, the number of scales is normally less than 10 which is tremendously small.

To simplify our problem, let us consider the following model

$$
\mathbf{Y}_{l}(t)=\dot{\mathbf{X}}_{l, 1}(t)+\dot{\mathbf{X}}_{l, 2}(t)+\sigma_{l} \mathbf{B}^{(1)}(t) .
$$

This model is similar with the original fMRI signal model defined in (1). If we successfully estimate the long memory parameters of the signals $\dot{\mathbf{X}}_{l, 1}$ and $\dot{\mathbf{X}}_{l, 2}$, we will be able to classify them into endogenous signal $\mathbf{X}_{l}$ and physiological noises (such as $\mathbf{C}_{l}$ or $\mathbf{R}_{l}$ ). From (26), the variance of filtered series of $\mathbf{Y}_{l}$ is

$$
\mathbf{E}\left(\overline{\left(\mathbf{Y}_{l}^{a^{j}}\right)^{2}}\right)=2^{2 d_{l, 1} j} \gamma_{l, 1}+2^{2 d_{l, 2} j} \gamma_{l, 2}+\sigma_{l}^{2}|a|^{2} .
$$

In the framework of the nonlinear least squares methods [1], our problem can be formulated as finding out the set of optimal parameters $\left(d_{l, 1}, d_{l, 2}, \gamma_{l, 1}, \gamma_{l, 2}, \sigma_{l}^{2}\right)$ which minimizes the least squares error $\chi_{L S}^{2}$ such that

$$
\chi_{L S}^{2}=\sum_{j=J_{1}}^{J_{2}}\left(2^{2 d_{l, 1} j} \gamma_{l, 1}+2^{2 d_{l, 2} j} \gamma_{l, 2}+\sigma_{l}^{2}|a|^{2}-\mathbf{E}\left(\overline{\left(\mathbf{Y}_{l}^{a^{j}}\right)^{2}}\right)\right)^{2}
$$

To solve the problem, we exploited the following simple algorithm. Let $\mathbb{D}:=$ $\left\{0, \frac{1}{r}, \frac{2}{r}, \cdots, 1\right\}$ be a discrete set of long memory parameters where $r$ is the resolution of long memory parameter. Then, we computed the following quantity for all $d_{l, 1} \in \mathbb{D}$ and $d_{l, 2} \in \mathbb{D}$

$$
\left(d_{l, 1}, d_{l, 2}\right)_{o p t}=\arg \min _{d_{l, 1}, d_{l, 2} \in \mathbb{D}} \chi_{L S}^{2}\left(d_{l, 1}, d_{l, 2}\right)
$$

where

$$
\begin{aligned}
\chi_{L S}^{2}\left(d_{l, 1}, d_{l, 2}\right)= & \sum_{j=J_{1}}^{J_{2}}\left[2^{2 d_{l, 1} j} \hat{\gamma}_{l, 1}\left(d_{l, 1}, d_{l, 2}\right)+2^{2 d_{l, 2} j} \hat{\gamma}_{l, 2}\left(d_{l, 1}, d_{l, 2}\right)\right. \\
& \left.+\hat{\sigma}_{l}^{2}\left(d_{l, 1}, d_{l, 2}\right)|a|^{2}-\mathbf{E}\left(\overline{\left(\mathbf{Y}_{l}^{a^{j}}\right)^{2}}\right)\right]^{2}
\end{aligned}
$$


and the vector $\mathbf{p}_{\text {opt }}=\left(\hat{\gamma}_{l, 1}\left(d_{l, 1}, d_{l, 2}\right), \hat{\gamma}_{l, 1}\left(d_{l, 1}, d_{l, 2}\right), \hat{\sigma}_{l}^{2}\left(d_{l, 1}, d_{l, 2}\right)\right)^{T}$ is determined by

$$
\mathbf{p}_{\text {opt }}=\arg \min _{\mathbf{p}}\|\mathbf{A p}-\mathbf{v}\|^{2}
$$

where

$$
\begin{aligned}
\mathbf{v}=\left[\mathbf{E}\left(\overline{\left(\mathbf{Y}_{l}^{a^{J_{1}}}\right)^{2}}\right),\right. & \left.\mathbf{E}\left(\overline{\left(\mathbf{Y}_{l}^{a^{J_{1}+1}}\right)^{2}}\right), \cdots, \mathbf{E}\left(\overline{\left(\mathbf{Y}_{l}^{a^{J_{2}}}\right)^{2}}\right)\right]^{T} \text { and } \\
\mathbf{A} & =\left[\begin{array}{ccc}
2^{2 d_{l, 1} J_{1}} & 2^{2 d_{l, 2} J_{1}} & 1 \\
2^{2 d_{l, 1}\left(J_{1}+1\right)} & 2^{2 d_{l, 2}\left(J_{1}+1\right)} & 1 \\
\vdots & \vdots & \vdots \\
2^{2 d_{l, 1} J_{2}} & 2^{2 d_{l, 2} J_{2}} & 1
\end{array}\right]
\end{aligned}
$$

given $d_{l, 1}$ and $d_{l, 2}[8]$.

\section{Experimental Results}

\subsection{Long Memory Processes Corrupted by White Noise}

To demonstrate that Achard et al.'s method [7] can be directly applied to general long memory processes, we simulated ARFIMA processes [9] contaminated by additive white noise since the process belongs to the class of long memory processes [6]. The process $\mathbf{X}$ is called the $\operatorname{ARFIMA}(p, d, q)$ process if $S^{*}(f)$ is given for $-1 / 2<d<1 / 2$ by

$$
S_{\mathbf{X}}^{*}(f)=\sigma^{2} \frac{\left|1+\sum_{k=1}^{q} \theta_{k} e^{-i f k}\right|^{2}}{\left|1-\sum_{k=1}^{p} \phi_{k} e^{-i f k}\right|^{2}}
$$

with $1-\sum_{k=1}^{p} \phi_{k} z^{k} \neq 0$ for $|z|=1[6]$.

To estimate the long memory parameter of the ARFIMA processes, we exploited the R-package dvfBm available on the R CRAN (http://cran.r-project. org). We will use the same notation as Achard et al. defined in [7]; that is, $\hat{H}^{S T}$ is a standard least squares estimator, $\hat{H}^{Q}$ is a least squares estimator based on sample quantiles, and $\hat{H}^{T M}$ is a least squares estimator based on trimmed mean. Also, $\hat{H}^{B 1-S T}, \hat{H}^{B 1-Q}$, and $\hat{H}^{B 1-T M}$ denote least squares estimators when the time series are assumed to be contaminated by white noise. Notice that the experimental results do not show the mean and standard deviation of the estimators since we did not run replication for each time series. We set up the specific parameters as follows: $\mathbf{p}=1 / 2$ and $\mathbf{c}=1$ for $\hat{H}^{Q}$, and $\beta_{1}=\beta_{2}=0.1$ for $\hat{H}^{T M}$. We only used the filter $i 1$ which corresponds to the increments of order 1 , and the minimum $M_{1}$ and the maximum $M_{2}$ of filter dilation were set up as 1 and 5 . The whole results are shown at the tables $1,2,3,4,5,6,7$, and 8. 


\begin{tabular}{|c|c|c|c|c|c|c|}
\hline & \multicolumn{3}{|c|}{$\mathrm{d}=0.2$} & \multicolumn{3}{c|}{$\mathrm{d}=0.4$} \\
\cline { 2 - 7 } & $\mathrm{n}=100$ & $\mathrm{n}=1000$ & $\mathrm{n}=10000$ & $\mathrm{n}=100$ & $\mathrm{n}=1000$ & $\mathrm{n}=10000$ \\
\hline $\mathrm{ST}(\mathrm{i} 1,5)$ & 0.160 & 0.172 & 0.159 & 0.442 & 0.374 & 0.366 \\
\hline Q(i1,5) & 0.266 & 0.155 & 0.159 & 0.512 & 0.349 & 0.365 \\
\hline TM(i1,5) & 0.174 & 0.173 & 0.158 & 0.495 & 0.355 & 0.366 \\
\hline
\end{tabular}

Table 1: Long memory estimates of an ARFIMA $(0, d, 0)$ process for $d=0.2,0.4$.

\begin{tabular}{|c|c|c|c|c|c|c|}
\hline & \multicolumn{3}{|c|}{$\mathrm{d}=0.2$} & \multicolumn{3}{c|}{$\mathrm{d}=0.4$} \\
\cline { 2 - 7 } & $\mathrm{n}=100$ & $\mathrm{n}=1000$ & $\mathrm{n}=10000$ & $\mathrm{n}=100$ & $\mathrm{n}=1000$ & $\mathrm{n}=10000$ \\
\hline $\mathrm{ST}(\mathrm{i} 1,5)$ & 0.337 & 0.304 & 0.290 & 0.463 & 0.555 & 0.530 \\
\hline $\mathrm{Q}(\mathrm{i} 1,5)$ & 0.397 & 0.283 & 0.280 & 0.402 & 0.550 & 0.527 \\
\hline $\mathrm{TM}(\mathrm{i} 1,5)$ & 0.364 & 0.310 & 0.283 & 0.456 & 0.558 & 0.529 \\
\hline
\end{tabular}

Table 2: Long memory estimates of an ARFIMA $(1, d, 0)$ process with $\phi_{1}=-0.2$ for $d=0.2,0.4$.

\begin{tabular}{|c|c|c|c|c|c|c|}
\hline & \multicolumn{3}{|c|}{$\mathrm{d}=0.2$} & \multicolumn{3}{c|}{$\mathrm{d}=0.4$} \\
\cline { 2 - 7 } & $\mathrm{n}=100$ & $\mathrm{n}=1000$ & $\mathrm{n}=10000$ & $\mathrm{n}=100$ & $\mathrm{n}=1000$ & $\mathrm{n}=10000$ \\
\hline $\mathrm{ST}$ & 0.050 & 0.026 & 0.016 & 0.097 & 0.203 & 0.185 \\
\hline $\mathrm{Q}$ & 0.105 & 0.031 & 0.015 & 0.208 & 0.210 & 0.179 \\
\hline $\mathrm{TM}$ & 0.090 & 0.036 & 0.019 & 0.114 & 0.195 & 0.182 \\
\hline
\end{tabular}

Table 3: Long memory estimates of an ARFIMA $(0, d, 1)$ process with $\theta_{1}=-0.3$ for $d=0.2,0.4$.

\begin{tabular}{|c|c|c|c|c|c|c|}
\hline & \multicolumn{3}{|c|}{$\mathrm{d}=0.2$} & \multicolumn{3}{c|}{$\mathrm{d}=0.4$} \\
\cline { 2 - 7 } & $\mathrm{n}=100$ & $\mathrm{n}=1000$ & $\mathrm{n}=10000$ & $\mathrm{n}=100$ & $\mathrm{n}=1000$ & $\mathrm{n}=10000$ \\
\hline $\mathrm{ST}$ & 1.718 & 1.686 & & 1.718 & 1.686 & \\
\hline $\mathrm{Q}$ & 2.155 & 2.149 & & 2.128 & 2.150 & \\
\hline $\mathrm{TM}$ & 1.971 & 1.942 & & 1.971 & 1.942 & \\
\hline
\end{tabular}

Table 4: Long memory estimates of an ARFIMA $(10, d, 0)$ process with $\phi_{i}=-0.2$ for $d=0.2,0.4$ and $i=1, \cdots, 10$. 


\begin{tabular}{|c|c|c|c|c|c|c|}
\hline & \multicolumn{3}{|c|}{$\mathrm{d}=0.2$} & \multicolumn{3}{c|}{$\mathrm{d}=0.4$} \\
\cline { 2 - 7 } & $\mathrm{n}=100$ & $\mathrm{n}=1000$ & $\mathrm{n}=10000$ & $\mathrm{n}=100$ & $\mathrm{n}=1000$ & $\mathrm{n}=10000$ \\
\hline $\mathrm{ST}$ & 0.117 & 0.279 & 0.277 & 0.529 & 0.539 & 0.556 \\
\hline $\mathrm{Q}$ & 0.107 & 0.321 & 0.272 & 0.445 & 0.497 & 0.553 \\
\hline TM & 0.163 & 0.292 & 0.280 & 0.511 & 0.532 & 0.562 \\
\hline
\end{tabular}

Table 5: Long memory estimates of an ARFIMA $(0, d, 10)$ process with $\theta_{i}=-0.3$ for $d=0.2,0.4$ and $i=1, \cdots, 10$.

\begin{tabular}{|c|c|c|c|c|c|c|}
\hline & \multicolumn{3}{|c|}{$\mathrm{d}=0.2$} & \multicolumn{3}{c|}{$\mathrm{d}=0.4$} \\
\cline { 2 - 7 } & $\mathrm{n}=100$ & $\mathrm{n}=1000$ & $\mathrm{n}=10000$ & $\mathrm{n}=100$ & $\mathrm{n}=1000$ & $\mathrm{n}=10000$ \\
\hline ST & 0.097 & 0.035 & 0.022 & -0.041 & 0.041 & 0.052 \\
\hline B1-ST & 0.049 & 0.297 & -0.182 & 0.271 & -0.360 & 0.148 \\
\hline B1-Q & 0.303 & -0.497 & -0.283 & 0.319 & 0.354 & 0.083 \\
\hline B1-TM & -0.032 & 0.238 & -0.143 & 0.212 & 0.472 & 0.086 \\
\hline
\end{tabular}

Table 6: Long memory estimates of an ARFIMA $(0, d, 0)$ process contaminated by white noise with a $S N R=-10$ for $d=0.2,0.4$.

\begin{tabular}{|c|c|c|c|c|c|c|}
\hline & \multicolumn{3}{|c|}{$\mathrm{d}=0.2$} & \multicolumn{3}{c|}{$\mathrm{d}=0.4$} \\
\cline { 2 - 7 } & $\mathrm{n}=100$ & $\mathrm{n}=1000$ & $\mathrm{n}=10000$ & $\mathrm{n}=100$ & $\mathrm{n}=1000$ & $\mathrm{n}=10000$ \\
\hline ST & 0.015 & 0.021 & 0.007 & 0.035 & 0.002 & 0.005 \\
\hline B1-ST & 0.056 & 0.004 & -0.255 & 0.014 & 0.415 & 0.539 \\
\hline B1-Q & -0.095 & 0.330 & -0.326 & 0.213 & 0.430 & -0.225 \\
\hline B1-TM & 0.106 & 0.330 & -0.546 & 0.336 & 0.683 & 0.324 \\
\hline
\end{tabular}

Table 7: Long memory estimates of an ARFIMA $(0, d, 0)$ process contaminated by white noise with a $S N R=-20$ for $d=0.2,0.4$.

\begin{tabular}{|c|c|c|c|c|c|c|}
\hline & \multicolumn{3}{|c|}{$\mathrm{d}=0.2$} & \multicolumn{3}{c|}{$\mathrm{d}=0.4$} \\
\cline { 2 - 7 } & $\mathrm{n}=100$ & $\mathrm{n}=1000$ & $\mathrm{n}=10000$ & $\mathrm{n}=100$ & $\mathrm{n}=1000$ & $\mathrm{n}=10000$ \\
\hline ST & 0.026 & -0.027 & 0.005 & 0.005 & 0.020 & 0.019 \\
\hline B1-ST & -0.502 & 0.238 & 0.203 & -0.363 & -0.059 & 0.880 \\
\hline B1-Q & -0.349 & -0.384 & 0.599 & -0.089 & 0.215 & 1.581 \\
\hline B1-TM & -0.581 & -0.093 & 0.324 & -0.525 & -0.172 & 0.726 \\
\hline
\end{tabular}

Table 8: Long memory estimates of an ARFIMA $(0, d, 10)$ process contaminated by white noise with a $S N R=-20$ and $\theta_{i}=-0.3$ for $d=0.2,0.4$ and $i=1, \cdots, 10$. 


\subsection{Linearly Summed fractional Brownian Motions}

We tested the NLS long memory estimator of linearly summed fractional Brownian motions with the Hurst parameter $H_{1}=0.1,0.2$ and $H_{2}=0.6,0.7,0.8,0.9$. Each process was simulated by the method proposed by Abry and Sellan [10]. (The MATLAB function can be found at http://www . mathworks.com/access / helpdesk/help/toolbox/wavelet/wfbm.html) To compute wavelet variances, we exploited the maximum overlay discrete wavelet transform (MODWT) with the Daubechies filter of order 8 . The results are shown at the table 9.

\begin{tabular}{|c|c|c|c|c|c|c|c|}
\hline \multicolumn{2}{|c|}{} & \multicolumn{3}{|c|}{$d_{1}=0.1$} & \multicolumn{3}{c|}{$d_{1}=0.2$} \\
\cline { 2 - 8 } \multicolumn{2}{|c|}{} & $\mathrm{n}=100$ & $\mathrm{n}=1000$ & $\mathrm{n}=10000$ & $\mathrm{n}=100$ & $\mathrm{n}=1000$ & $\mathrm{n}=10000$ \\
\hline \multirow{2}{*}{$d_{2}=0.6$} & $\hat{d}_{1}$ & 0.000 & 0.002 & 0.003 & & 0.003 & 0.002 \\
\cline { 2 - 8 } & $\hat{d}_{2}$ & 1.000 & 0.538 & 0.355 & & 0.212 & 0.532 \\
\hline \multirow{2}{*}{$d_{2}=0.7$} & $\hat{d}_{1}$ & 0.000 & 0.003 & 0.002 & 0.000 & 0.000 & 0.002 \\
\cline { 2 - 8 } & $\hat{d}_{2}$ & 1.000 & 0.422 & 0.483 & 1.050 & 0.939 & 0.565 \\
\hline \multirow{2}{*}{$d_{2}=0.8$} & $\hat{d}_{1}$ & 0.000 & 0.002 & 0.002 & 0.000 & 0.002 & 0.365 \\
\cline { 2 - 8 } & $\hat{d}_{2}$ & 1.000 & 0.644 & 0.608 & 1.000 & 0.593 & 1.235 \\
\hline \multirow{2}{*}{$d_{2}=0.9$} & $\hat{d}_{1}$ & 0.000 & 0.001 & 0.001 & 0.000 & 0.000 & 0.002 \\
\cline { 2 - 8 } & $\hat{d}_{2}$ & 1.000 & 0.848 & 0.721 & 1.000 & 1.319 & 0.557 \\
\hline
\end{tabular}

Table 9: Long memory estimates of two linearly summed fractional Brownian motions for $H_{1}=0.1,0.2$, and $H_{2}=0.6,0.7,0.8,0.9$.

\section{Discussion}

Estimation of long memory parameters in the presence of white noise

We applied Achard et al.'s method [7] to the $\operatorname{ARFIMA}(p, d, q)$ processes corrupted by white noises, but the performance was extremely poor except the case of $\operatorname{ARFIMA}(0, d, 0)$. We thought that the applicability of Achard et al.'s method to a long memory process $\mathbf{X}$ is based on the assumption such that $S_{\mathbf{X}}^{*}(f)$ of the process $\mathbf{X}$ belongs to the function set $\mathcal{H}(\beta, L)$ defined at $(8)$. The fact that the parameter estimation of the $\operatorname{ARFIMA}(0, d, 10)$ process was better than that of the $\operatorname{ARFIMA}(0, d, 0)$ process implies that $S_{\mathbf{X}}^{*}(f)$ better fit into $\mathcal{H}(\beta, L)$ as the order $q$ of MA part increases. Nevertheless, we need to understand exactly why the performance of estimation was significantly unacceptable, and to enhance Achard et al.'s method in order to make better performance for general long memory processes.

\section{Parameter estimation of linearly combined long memory processes}

The parameter estimation of linearly combined long memory processes was seriously worse than that of a single long memory processes corrupted by white 
noise. As you see in the table 9, the estimates are not contiguous to the expected value. We need to verify how the NLS-based estimator is effective theoretically and empirically. As I noticed at the section 4, the small number of scales seems to contribute to the poor performance. In the future works, we will clarify what factors mostly influence the performance of estimation. In addition, we will attempt to develop the alternative methods for parameter estimation of linearly combined long memory processes.

\section{References}

1. A. Istratov, O. Vyvenko, Exponential analysis in physical phenomena, Review Of Scientific Instruments. 70 (1999) 1233.

2. G.L. Bretthorst, W.C. Hutton, J.R. Garbow, J.J. Ackerman, Exponential parameter estimation (in NMR) using Bayesian probability theory, Concepts In Magnetic Resonance Part A. 27A (2005) 55-63.

3. E. Bullmore, J. Fadili, V. Maxim, L. Sendur, B. Whitcher, J. Suckling, et al., Wavelets and functional magnetic resonance imaging of the human brain., NeuroImage. 23 Suppl 1 (2004) S234-49.

4. A. Wink, E. Bullmore, A. Barnes, F. Bernard, J. Suckling, Monofractal and multifractal dynamics of low frequency endogenous brain oscillations in functional MRI, Human Brain Mapping. 29 (2008) 791-801.

5. E. Moulines, F. Roueff, M. Taqqu, On the Spectral Density of the Wavelet Coefficients of Long-Memory Time Series with Application to the Log-Regression Estimation of the Memory Parameter, Journal Of Time Series Analysis. 28 (2007) 155187.

6. S. Achard, D.S. Bassett, A. Meyer-Lindenberg, E. Bullmore, Fractal connectivity of long-memory networks, Physical Review E. 77 (2008) 1-12.

7. S. Achard, J. Coeurjolly, Discrete variations of the fractional Brownian motion in the presence of outliers and an additive noise, Statistics Surveys. (2009) 1-26.

8. Lawson, C.L. and R.J. Hanson, Solving Least Squares Problems, Prentice-Hall, 1974, Chapter 23, p. 161.

9. Simone Fatichi, ARFIMA simulations, MATLAB Central, 2009. http://www.mathworks.com/matlabcentral/fileexchange/25611

10. Abry, P.; F. Sellan (1996), The wavelet-based synthesis for the fractional Brownian motion proposed by F. Sellan and Y. Meyer: Remarks and fast implementation, Appl. and Comp. Harmonic Anal., 3(4), pp. 377-383.

11. J.B. Bassingthwaighte, L.S. Liebovitch, B.J. West, Fractal physiology, Oxford University Press US, 1994.

12. V. Maxim, L. Şendur, J. Fadili, J. Suckling, R. Gould, R. Howard, et al., Fractional Gaussian noise, functional MRI and Alzheimer's disease, NeuroImage. 25 (2005) 141158.

13. R.M. Birn, J.B. Diamond, M.A. Smith, P.A. Bandettini, Separating respiratoryvariation-related fluctuations from neuronal-activity-related fluctuations in fMRI, NeuroImage. 31 (2006) 1536-48.

14. D. Cordes, V.M. Haughton, K. Arfanakis, J.D. Carew, P.a. Turski, C.H. Moritz, et al., Frequencies contributing to functional connectivity in the cerebral cortex in "resting-state" data., AJNR. American Journal Of Neuroradiology. 22 (2001) 1326-33. 
15. M. Kobayashi, T. Musha, 1/f Fluctuation of Heartbeat Period, IEEE Transactions On Bio-medical Engineering. 29 (1982) 456-7.

16. C. Peng, S. Havlin, J. Hausdorff, J. Mietus, H. Stanley, A. Goldberger, Fractal mechanisms and heart rate dynamics: Long-range correlations and their breakdown with disease, Journal Of Electrocardiology. 28 (1995) 59-65.

17. P.C. Ivanov, L.A. Amaral, A.L. Goldberger, S. Havlin, M.G. Rosenblum, Z.R. Struzik, et al., Multifractality in human heartbeat dynamics, Nature. 399 (1999) 461-5.

18. A.L. Goldberger, Fractal Electrodynamics of the Heartbeat, Annals Of The New York Academy Of Sciences. 591 (1990) 402-409.

19. B. West, R. Zhang, A. Sanders, S. Miniyar, J. Zuckerman, B. Levine, Fractal fluctuations in cardiac time series, Physica A: Statistical Mechanics And Its Applications. 270 (1999) 552-566.

20. Y. Yamamoto, Y. Nakamura, H. Sato, M. Yamamoto, K. Kato, R.L. Hughson, On the fractal nature of heart rate variability in humans: effects of vagal blockade, The American Journal Of Physiology. 269 (1995) R830-7.

21. G. DAddio, A. Accardo, G. Corbi, F. Rengo, Fractal analysis of heart rate variability in COPD patients, Berlin, Heidelberg, Springer Berlin Heidelberg, 2007.

22. P.J. Fadel, S.M. Barman, S.W. Phillips, G.L. Gebber, Fractal fluctuations in human respiration, Journal Of Applied Physiology (Bethesda, Md. : 1985). 97 (2004) 205664.

23. C. Peng, J.E. Mietus, Y. Liu, C. Lee, J.M. Hausdorff, H.E. Stanley, et al., Quantifying Fractal Dynamics of Human Respiration: Age and Gender Effects, Annals Of Biomedical Engineering. 30 (2002) 683-692.

24. H.H. Szeto, P.Y. Cheng, J.A. Decena, Y. Cheng, D.L. Wu, G. Dwyer, Fractal properties in fetal breathing dynamics, The American Journal Of Physiology. 263 (1992) R141-7.

25. C. Thäle, Further Remarks on Mixed Fractional Brownian Motion, Applied Mathematical Sciences. 3 (2009) 18851901.

26. Yaglom, A. M., Correlation theory of processes with random stationary nth increments. American Mathematical Society Translation (2) 8 (1958) 87141.

27. J. Coeurjolly, Estimating the parameters of a fractional Brownian motion by discrete variations of its sample paths, Statistical Inference For Stochastic Processes. 4 (2001) 199227. 